\title{
Machine Learning: Discovering the Future of Medical Imaging
}

\author{
Bradley J. Erickson ${ }^{1}$ (D)
}

Published online: 26 June 2017

(C) Society for Imaging Informatics in Medicine 2017

Machine learning, and deep learning in particular, is seen as both the hope and the downfall of the medical imaging profession. Some hail the promise of computers reliably identifying each and every finding, and some decry it as the demise of medicine. This issue is devoted to reports on the application of machine learning in medical imaging, some of which were presented at the inaugural Conference on Machine Intelligence in Medical Imaging (C-MIMI).

"With Great Power Comes Great Responsibility"Spiderman

This issue of JDI includes a broad range of manuscripts including ones describing segmentation, classification, or diagnosis using MR, CT, and US images. There are papers describing techniques that are broadly useful in machine learning, and some review articles that provide a vision of the landscape of machine learning in medical imaging. The breadth of articles and the techniques described is impressive and reflects that we are in the early development stages of the application of machine learning in medical imaging.

At the same time, we must be careful that the methods applied and the findings reported are reliable. Machine learning and deep learning, in particular, have great power to find things that may not be important but seem to be. Spurious connections are a constant risk for the machine learning scientist, and multiple rigorous tests must be applied to be certain that the apparent capability of an algorithm is correct.

"Everything In Life Is Speaking Despite Its Apparent Silence"-Hazrat Khan

Bradley J. Erickson

bje@mayo.edu

1 Department of Radiology, Mayo Clinic, Rochester, MN 55905, USA
Machine learning does have tremendous promise to reliably identify many findings in images. While "Computers Replacing Radiologists" has been a common focus in the popular press, what may be the most exciting is that machine learning seems to be able to find features in images that are not apparent to humans; included in this issue are manuscripts describing prediction of genomic and molecular properties that were once thought only identifiable with tissue. These capabilities are the drivers behind the great excitement and investment in this new field.

It is incumbent upon those of us in this field to understand and appreciate the potential of these methods to discover things about medical images that may not be intuitive or expected. This "Discovery Science" is an area that has not been embraced in medical imaging but is a core value in genomics and precision medicine. Medical imaging must also take up the "Discovery Science" flag and recognize that important advances are not always based on hypothesis testing.

"The measure of a superhero is always his nemesis"David Lyons

This special issue demonstrates the incredible potential of machine learning to advance medical imaging science, and the attention to methods also demonstrates how we must avoid the pitfalls. Machine learning is already dramatically altering the everyday life of everyone and everything on the planet. Weather predictions, self-driving cars, satellite-guided crop husbandry and of course, medical imaging.

Medical imaging has tremendous resources at its disposal. The Biden Cancer Moonshot specifically identifies the tremendous assets that are already available to be studied, and the need to efficiently convert the information present into knowledge. We have a responsibility to use those resources to assure that our patients receive the best care possible in the most costeffective way. Machine learning and deep learning in particular, appears to be an important component of achieving that goal. 